\title{
FACTORES QUE INFLUYEN EN LA TOMA DE DECISIONES EN ESTUDIANTES DE SECUNDARIA
}

\section{FACTORS INFLUENCING DECISION MAKING IN HIGH SCHOOL STUDENTS}

\author{
Odacir HERNÁNDEZ ALMANZA ${ }^{1}$
}

\begin{tabular}{|lll|}
\hline Recibido & $:$ & 26.11 .2020 \\
Aceptado & $:$ & 17.12 .2020 \\
Publicado & $:$ & 12.02 .2021 \\
\hline
\end{tabular}

\begin{abstract}
RESUMEN: La toma de decisiones (TD) es una respuesta ante un estímulo que podría o no, repercutir en la vida del sujeto y su entorno. Siendo así, esta investigación tiene como objetivo, describir la TD, como actividad fundamental en la caracterización de los estudiantes de secundaria, teniendo en cuenta el conocimiento y el sentimiento o afectividad, aplicados en situaciones de estrés, familiares, pares e interés propio. La metodología corresponde al enfoque cuantitativo con un alcance descriptivo. Se aplicó un cuestionario a los estudiantes del Colegio Aquileo Parra IED de Bogotá, utilizando las escalas Likert y Osgood. Los resultados muestran coherencia con estudios anteriores que establecen la independencia del estudiante en la TD al ir avanzando en edad y en los ciclos de enseñanza. Sin embargo, refleja un bajo porcentaje de satisfacción en la TD por parte de los estudiantes, mostrando influencias fuertes de los pares y debilidades con el estrés. Esto explica los resultados negativos en lo convivencial y académico. La investigación aporta datos a la caracterización, y posibilita la realización de un nuevo estudio que incluya, acciones sobre el afianzamiento de la habilidad de TD en el currículo.
\end{abstract}

Palabras clave: toma de decisiones, habilidad, conocimiento, actitud, sentimiento.

\begin{abstract}
Decision Making (DM) is a response to a stimulus that might or may not affect the life of the subject and its environment. Thus, this research evaluates DM, as a fundamental activity in the characterization of secondary students, taking into account the knowledge and feeling or affection, applied in situations of stress, family, peers and self-interest. The methodology corresponds to the quantitative approach with a descriptive scope. A questionnaire was applied to the students of Aquileo Parra School IED in Bogotá, using the scales Likert and Osgood. The result showed coherence with previous studies that establish the independence of the student in the DM when advancing in age and cycles. However, it reflects a low percentage of DM satisfaction on the part of students, showing torn peer influences and stress weaknesses. This explains the negative results in their convivial and academic a lives. The Research provides data to the characterization, and makes possible the realization of a new study that includes, actions on the reinforcement of the ability of DM in the curriculum.
\end{abstract}

Keywords: Decision making, skill, knowledge, attitude, feeling.

\footnotetext{
${ }^{1}$ Magister en Educación. Colegio Aquileo Parra IED. Bogotá D.C-Colombia. Email: hodacir@yahoo.com. ORCID: https://orcid.org/0000-0002-2849-7579
} 


\section{Journal of the Academy $|43|$}

\section{INTRODUCCIÓN}

El estudio de las relaciones de las competencias emocionales y la habilidad de la toma de decisiones (TD), ha tenido alto impacto en los últimos años. Desde este punto de vista Márquez-Cervantes y Gaeta-González (2018), plantean que los padres y profesores como coeducadores, tienen un trabajo para prevenir que los chicos sean afectados por situaciones de riesgo. La TD forma parte de las Habilidades para la Vida que, desde la División de Salud Mental de la Organización Mundial de la Salud, se impulsa en un documento ajustado en 1997, donde se sugiere poner en práctica el programa en centros educativos, Martínez (2014). De este modo, se encuentra en congruencia con las políticas públicas y educativas del Ministerio de Educación Nacional MEN, desde la ley 115 de 1994, reglamentada en el decreto 1860 de 1994, y en la ley 1620 de 2013.

Las Habilidades para la Vida según Martínez (2014), se enmarcan en el desarrollo de competencias humanas que se limita al manejo de algunas técnicas psicosociales. La TD tiene relevancia cuando González, Guevara, Jiménez y Alcázar (2018), señalan la implicación con otras habilidades como la asertividad, pueden estar asociadas al rendimiento académico y a la ansiedad en los estudiantes. En este sentido, el estudio de Vieira, Barbosa y Goncalves (2018), considera que las características bioecológicas (habilidades sociales) y el contexto (estrés en la escuela), influyen en el rendimiento académico en los chicos de sexto grado. El contexto de la estructura familiar es relevante cuando Lastra, Durán y Acosta (2018), enfatizan en los ambientes favorables de las familias para mejorar el rendimiento académico y la convivencia de los estudiantes.

Con lo anterior, el presente estudio desarrolla un planteamiento teórico sobre los factores que influyen en la TD. Luego, aplica una metodología con enfoque cuantitativo y con carácter descriptivo, que mide la capacidad de la TD según factores planteados en la parte teórica. Seguidamente, se analiza los resultados teniendo en cuenta tablas estadísticas sobre el rendimiento académico y convivencial, finalizando con la discusión de resultados y las conclusiones. 


\section{Journal of the Academy $|44|$}

\section{DESARROLLO}

\section{El conocimiento y la emotividad en la Toma de Decisiones.}

La caracterización de estudiantes implica el conocimiento de las condiciones socioeconómicas y psicosociales que posee el sujeto para aplicar la TD, con el fin de dar solución a las necesidades particulares y del entorno. Por lo anterior, la TD se considera como una habilidad social que integra al sujeto a la comunidad. El estudio elaborado por Cote y García (2016), establece una relación directa entre, el proceso cognitivo, las interconexiones neuronales, el estrés y el género, como factores que intervienen en la TD y que afectan la evaluación de las alternativas que ofrece situación y que perjudican los intereses y la conducta de una persona. Por otro lado, Portillo (2017) considera que la educación por habilidades es una construcción social que depende del contexto y los intereses del sujeto. Además, que "la autoestima y la habilidad de toma de decisiones son más complejas y que requieren de una serie de procesos y etapas previas, así como una mayor temporalidad para su desarrollo" (Choque y Chirinos, 2009, p. 178). En este sentido, el estudio de Enciso y Lozano (2011), mide actitudes y estrategias de pensamiento social, que contribuyen al desarrollo de actitudes y estrategias cognitivas para fortalecer la competencia social. A su vez, Mora, Martínez y Serrano (2013), afirman que las actitudes se determinan por los componentes cognitivo, afectivo y conductual, que se deben operacionalizar para realizar una evaluación de la habilidad de la TD. En otra asociación, el modelo bioecológico del desarrollo humano, señala que los principales componentes del modelo "el Proceso, la Persona, el Contexto y el Tiempo, contribuyen a la comprensión del desarrollo de las habilidades sociales a lo largo de la vida de la persona" (Barbosa, Pereira y Kopller, 2015, p.187), es decir, la familia, la escuela y el trabajo.

Por otro lado, los estudios realizados por Cote y García (2016); Vargas, Henao y González (2007); Gualano de Godoy (2011), han establecido que las condiciones como el estrés, el ámbito familiar, la edad y los propios intereses, son importantes para tener en cuenta en el momento de la TD.

Para enfocar el estudio, la TD se presenta como la respuesta a un estímulo producto de la combinación de otras habilidades. Se arguye, que fortalecer la TD, los procesos cognitivos, emocionales y afectivos, para mejorar la competencia psicosocial, es un trabajo que demanda tiempo, Choque y Chirinos (2009). En este sentido, Enciso y Lozano (2011) en su estudio de 


\section{Journal of the Academy | $45 \mid$}

medición de actitudes y estrategias cognitivas de actitudes sociales, señalan la necesidad de estimular programas que desarrollen actitudes y estrategias cognitivas para mejorar la competencia social. De este modo, "los escenarios familiares y centros infantiles, bien nombrados espacios de socialización, son altamente favorables para el acceso de los niños a los códigos sociales" (Gallego, 2015, p. 102). Igualmente, Martínez, Tovar, Rojas y Duque (2008), establecieron la importancia de la crianza con efectos directos en las actitudes sociales en estudiantes y cuidadores. En consecuencia, los espacios colectivos son herramientas para la adquisición de conocimiento a través de la experiencia y posteriormente, utilizarlo en la TD.

Por otro lado, en los espacios colectivos, encontramos diferencias interculturales que hay que tener en cuenta en el momento de medir las actitudes porque dependen de la intensidad de las emociones, según el conocimiento y las experiencias vividas en la cultura, Moscoso y Spielberger (2011). Entonces, las emociones pueden interferir en la TD. Desde este punto de vista, Bonilla, González, Bonilla-Santos, Muñoz y Baldovino (2017), establece en su estudio que los niños y niñas que se encuentran en riesgo, poseen menor rendimiento en la TD. Igualmente, señala la existencia de los marcadores somáticos establecidos en el sujeto por experiencias pasadas que marcan la forma de respuesta visceral a un estímulo negativo.

Otro de los factores que influye en la TD es el estrés. "Existe una relación negativa entre el estrés y la TD; que esta relación no solo provoca cambios comportamentales (irritabilidad, aislamiento o depresión) ...” (Cote y García, 2016, p. 25). En este estudio, se encontró una relación inversa entre el estrés y la TD, a mayor estrés, menor es la capacidad de respuesta efectiva a través de la TD. Lo anterior está ligado a la inteligencia emocional que desarrolla emociones correctas de índole intrapersonal e interpersonal, en momentos precisos para afrontar los retos de la vida, Barrantes (2016).

Con lo anterior, la adquisición de conocimiento y el manejo correcto de los sentimientos en la TD, aumenta con la edad. En el estudio de González, Orcasita, Carrillo y Palma (2017), encuentran que la TD en adolescentes sobre el manejo de su sexualidad, depende en gran parte del conocimiento y de la experiencia de los padres. Aquí, se evidencia cierta dependencia hacia los padres en el comienzo de la adolescencia. Posteriormente, al avanzar en edad, tienen en cuenta la opinión de sus pares y, por último, el criterio personal. En ese sentido, "en cuanto más ajustadas son las personas en sus relaciones personales y nivel cognitivo, mayor es el repertorio de habilidades sociales" (Di Domenico y Scortegagna, 2018, p. 8). 


\section{Journal of the Academy $|46|$}

Para complementar, se deben verificar situaciones vividas o conocidas en su contexto para medir la TD. En este caso, el estudio de Veloso, Magalhães, Vasconcelos, Costa, Teixeira y Rayane de Sampaio (2018), aplica un método para verificar las evidencias de validez del constructo, teniendo en cuenta parámetros psicométricos globales que se adecuan al contexto.

\section{Tabla 1}

Categorización Para Medir Conocimiento en la TD

\begin{tabular}{ll}
\hline Valor & Grado de conocimiento \\
\hline 0 & Decisiones impulsivas que no corresponden a la situación. \\
1 & Decisiones que corresponden parcialmente a la situación, pero que no son las esperadas. \\
2 & Decisiones que consideren algunas causas y algunas consecuencias. \\
3 & Decisiones que consideren todas las causas y todas las consecuencias. \\
\hline
\end{tabular}

Nota: Escala de grado de conocimiento. Elaboración propia.

Categorías para verificar las actitudes en la TD. Se utilizó un cuestionario con escala LIKERT. A continuación, se presenta en la tabla 2 la respectiva categorización

\section{Tabla 2}

Categorización Para Medir la Actitud en la TD

\begin{tabular}{ll}
\hline Valor & Actitud \\
\hline 0 & No posee actitud positiva ante las consideraciones sociales al tomar todas decisiones. \\
1 & Posee poca actitud positiva ante la consideración social al tomar algunas decisiones \\
2 & Posee parcialmente actitud positiva ante la consideración social al tomar decisiones \\
3 & Posee poca actitud positiva ante la consideración social al tomar decisiones \\
\hline
\end{tabular}

Nota: Escala de grado de actitud. Elaboración propia

Categorías para medir el grado de emotividad en la TD. Se utilizó un cuestionario con escala OSGOOD. A continuación, se presenta la tabla 3 con la respectiva categorización. 


\section{Journal of the Academy $|47|$}

\section{Tabla 3}

Categorización Para Medir Emotividad en la TD

\begin{tabular}{ll}
\hline Valor & Grado de emotividad \\
\hline 1 & Su percepción emotiva, es nula ante la toma de decisión \\
2 & Su percepción emotiva, es parcialmente positiva ante la toma de algunas decisiones \\
3 & Su percepción emotiva, es parcialmente positiva ante la toma de decisiones \\
4 & Su percepción emotiva, es positiva ante la mayoría toma de decisiones
\end{tabular}

Nota: Escala de grado de emotividad. Elaboración propia

\section{RESULTADOS}

Los datos socioeconómicos de la población muestran, indican una ubicación en el estrato tres del $57.1 \%$; en el estrato dos del $32.2 \%$; en el estrato uno del $1.5 \%$ y en el estrato cuatro del $8 \%$. Se encuentra que el $17.6 \%$ de papás, no poseen estudio. El $25.3 \%$ tienen estudio de básica primaria. El $23.8 \%$ no terminaron los estudios de secundaria y el $23.8 \%$ de padres, terminaron su bachillerato. Finalmente, un promedio del $10 \%$ de papás, poseen estudios técnicos o universitarios. Importante destacar que el $8.8 \%$ de los estudiantes trabaja para su sustento diario y que el $51 \%$, los padres son separados.

En la medición del Conocimiento, actitudes y emotividad en la TD se encontró:

\section{Medición de conocimiento en la TD.}

Los resultados se presentan por ciclos de enseñanza (III, IV y V), teniendo en cuenta las subcategorías de situaciones de estrés, familia, pares e intereses personales. Se establecen las mediciones basadas en la media y en la moda para cada pregunta con el fin de destacar las debilidades y las fortalezas en la TD. Para la medición tenemos en cuenta los resultados relevantes en las siguientes situaciones:

A. Estrés: $2=$ perder el trabajo; $3=$ presentar recuperaciones; $4=$ amenaza 


\section{Journal of the Academy $|48|$}

B. Familiar: $5=$ escoger carrera; $6=$ modelo de hermano; $7=$ Noviazgo $; 8=$ cumplir con el estudio.

C. Pares: $9=$ en contra de un profesor; $10=$ presión para fumar; $11=$ Presión para jugar; $12=$ participación en el colegio

D. Interés personal: 13= aceptar beca; 14= comprensión de temas; 15= participar campeonato; $16=$ proyecto de vida

\section{Tabla 4}

Estadística medición del conocimiento en la toma de decisiones

\begin{tabular}{|c|c|c|c|c|c|c|c|c|c|c|c|c|c|c|c|c|}
\hline & & \multicolumn{3}{|c|}{ Estrés } & \multicolumn{4}{|c|}{ Familia } & \multicolumn{4}{|l|}{ Pares } & \multicolumn{4}{|c|}{ Interés personal } \\
\hline & & 2 & 3 & 4 & 5 & 6 & 7 & 8 & 9 & 10 & 11 & 12 & 13 & 14 & 15 & 16 \\
\hline $\mathrm{C}$ & Válido & 111 & 111 & 111 & 111 & 111 & 111 & 111 & 111 & 111 & 111 & 111 & 111 & 111 & 111 & 111 \\
\hline $\begin{array}{l}\mathrm{i} \\
\mathrm{c}\end{array}$ & $\begin{array}{l}\text { Perdido } \\
\mathrm{s}\end{array}$ & 0 & 0 & 0 & 0 & 0 & 0 & 0 & 0 & 0 & 0 & 0 & 0 & 0 & 0 & 0 \\
\hline $\begin{array}{l}1 \\
0\end{array}$ & Media & 1,63 & 2,32 & $\begin{array}{l}2,6 \\
4\end{array}$ & 2,43 & 1,54 & 2,23 & 1,77 & 1,90 & 2,60 & 2,67 & 2,03 & 1,92 & 2,02 & 2,26 & 2,59 \\
\hline I & $\begin{array}{l}\text { Median } \\
\text { a }\end{array}$ & 1,00 & 3,00 & $\begin{array}{l}3,0 \\
0\end{array}$ & 3,00 & 2,00 & 3,00 & 2,00 & 2,00 & 3,00 & 3,00 & 3,00 & 2,00 & 2,00 & 2,00 & 3,00 \\
\hline I & $\begin{array}{l}\text { Moda } \\
\text { Desv. }\end{array}$ & $\begin{array}{l}1 \\
, 981\end{array}$ & $\begin{array}{l}3 \\
, 822\end{array}$ & $\begin{array}{l}3 \\
, 74 \\
8\end{array}$ & $\begin{array}{l}3 \\
, 940\end{array}$ & $\begin{array}{l}2 \\
1,15\end{array}$ & $\begin{array}{l}3 \\
1,09\end{array}$ & $\begin{array}{l}2 \\
, 963\end{array}$ & $\begin{array}{l}3 \\
1,13\end{array}$ & $\begin{array}{l}3 \\
, 664\end{array}$ & $\begin{array}{l}3 \\
, 790\end{array}$ & $\begin{array}{l}3 \\
1,21\end{array}$ & $\begin{array}{l}2 \\
, 811\end{array}$ & $\begin{array}{l}2 \\
, 763\end{array}$ & $\begin{array}{l}2 \\
, 747\end{array}$ & $\begin{array}{l}3 \\
, 792\end{array}$ \\
\hline $\begin{array}{l}\text { C } \\
\text { i } \\
\text { c }\end{array}$ & $\begin{array}{l}\text { Válido } \\
\text { Perdido } \\
\mathrm{s}\end{array}$ & $\begin{array}{l}74 \\
0\end{array}$ & $\begin{array}{l}74 \\
0\end{array}$ & $\begin{array}{l}74 \\
0\end{array}$ & $\begin{array}{l}74 \\
0\end{array}$ & $\begin{array}{l}74 \\
0\end{array}$ & $\begin{array}{l}74 \\
0\end{array}$ & $\begin{array}{l}74 \\
0\end{array}$ & $\begin{array}{l}74 \\
0\end{array}$ & $\begin{array}{l}74 \\
0\end{array}$ & $\begin{array}{l}74 \\
0\end{array}$ & $\begin{array}{l}74 \\
0\end{array}$ & $\begin{array}{l}74 \\
0\end{array}$ & $\begin{array}{l}74 \\
0\end{array}$ & $\begin{array}{l}74 \\
0\end{array}$ & $\begin{array}{l}74 \\
0\end{array}$ \\
\hline $\begin{array}{l}1 \\
0\end{array}$ & Media & 2,04 & 2,68 & $\begin{array}{l}2,6 \\
9\end{array}$ & 2,68 & 1,54 & 2,74 & 2,00 & 2,27 & 2,76 & 2,81 & 1,89 & 2,14 & 2,30 & 2,38 & 2,77 \\
\hline V & $\begin{array}{l}\text { Median } \\
\text { a }\end{array}$ & 2,00 & 3,00 & $\begin{array}{l}3,0 \\
0\end{array}$ & 3,00 & 2,00 & 3,00 & 2,00 & 3,00 & 3,00 & 3,00 & 3,00 & 2,00 & 2,00 & 2,50 & 3,00 \\
\hline & $\begin{array}{l}\text { Moda } \\
\text { Desv. }\end{array}$ & $\begin{array}{l}3 \\
, 898\end{array}$ & $\begin{array}{l}3 \\
, 622\end{array}$ & $\begin{array}{l}3 \\
, 75 \\
7\end{array}$ & $\begin{array}{l}3 \\
, 664\end{array}$ & $\begin{array}{l}2 \\
1,03\end{array}$ & $\begin{array}{l}3 \\
, 621\end{array}$ & $\begin{array}{l}1 \\
, 891\end{array}$ & $\begin{array}{l}3 \\
1,11\end{array}$ & $\begin{array}{l}3 \\
, 491\end{array}$ & $\begin{array}{l}3 \\
, 655\end{array}$ & $\begin{array}{l}3 \\
1,32\end{array}$ & $\begin{array}{l}2 \\
, 970\end{array}$ & $\begin{array}{l}3 \\
, 789\end{array}$ & $\begin{array}{l}3 \\
, 735\end{array}$ & $\begin{array}{l}3 \\
, 538\end{array}$ \\
\hline $\mathrm{C}$ & Válido & 75 & 75 & 75 & 75 & 75 & 75 & 75 & 75 & 75 & 75 & 75 & 75 & 75 & 75 & 75 \\
\hline $\begin{array}{l}\mathrm{i} \\
\mathrm{c}\end{array}$ & $\begin{array}{l}\text { Perdido } \\
\mathrm{s}\end{array}$ & 0 & 0 & 0 & 0 & 0 & 0 & 0 & 0 & 0 & 0 & 0 & 0 & 0 & 0 & 0 \\
\hline o & Media & 2,12 & 2,68 & $\begin{array}{l}2,3 \\
5\end{array}$ & 2,75 & 1,63 & 2,44 & 1,91 & 2,44 & 2,80 & 2,63 & 1,67 & 2,31 & 2,52 & 2,27 & 2,64 \\
\hline V & $\begin{array}{l}\text { Median } \\
\text { a }\end{array}$ & 2,00 & 3,00 & $\begin{array}{l}3,0 \\
0\end{array}$ & 3,00 & 2,00 & 3,00 & 2,00 & 3,00 & 3,00 & 3,00 & 3,00 & 3,00 & 3,00 & 2,00 & 3,00 \\
\hline & Moda & 3 & 3 & 3 & 3 & 2 & 3 & 1 & 3 & 3 & 3 & 3 & 3 & 3 & 3 & 3 \\
\hline
\end{tabular}


Journal of the Academy $\mid 49$ |

$\begin{array}{lllllllllllllll}\text { Desv. } & \text { 9584 }\end{array}$

Nota: Comparación de las medias en las situaciones de estrés, familiar, pares e interés personal, en los ciclos III, IV y V. Elaboración propia

El ciclo III, presenta una media en edad de 12.23 en un total de 111 estudiantes encuestados. En el ciclo IV, se encuestaron 74 estudiantes que presentan una media en edad de $14.39 \mathrm{y}$ en el ciclo $\mathrm{V}$, se encuestaron 75 estudiantes que presentan una media en edad de 16.24.

La tabla 4, muestra en situaciones de estrés, en el ciclo III, llama la atención la TD en el riesgo de perder el trabajo con una media de 1.63. Mientras en el ciclo IV se presenta una fortaleza con el 2.04 en la media y en el ciclo V, presenta mayor seguridad con el 2.12. Por otro lado, la TD en situación de amenaza, muestra medias respectivas 2.64 , en el ciclo III que es similar al ciclo IV con una media de 2.64 y menos seguridad en ciclo V con la media de 2. 35. Seguidamente, la TD en cuanto a la presentación de recuperaciones, el ciclo III muestra una media de 2,32 que es inferior a las de los ciclos IV y V que tienen una media de 2.68. Lo anterior, sugiere que los niños mayores aprovechan más las recuperaciones.

En las situaciones de influencia de la familia en el ciclo III, llama la atención que en la escogencia de carrera los estudiantes muestran una media de 2.43 , mostrando inseguridad con respecto al ciclo IV que muestra una media de 2,68 y este a su vez, inferior al ciclo V con una media de 2.75. Lo que indica mayor independencia en el ciclo V. En cuanto al manejo del noviazgo, los estudiantes del ciclo III, muestran una media 2.23 , mientras, los del ciclo IV muestran una media de 2.74 y los de ciclo $\mathrm{V}$, una media de 2.44. Lo anterior indica que los estudiantes de ciclo IV suelen ser más desprevenidos en el noviazgo y los de ciclos V, más controlados. En este grupo de preguntas, la TD en la situación de influencia de hermanos muestra en el ciclo III, una media de 1.54 y en los ciclos IV y V, una media de 2.0. Esto indica que los estudiantes consideran que no influyen en la TD de sus hermanos. Seguidamente, en el ciclo III, la influencia de los padres vs la responsabilidad escolar muestra una media de $1.77 \mathrm{y}$ en los ciclos IV y V de 2.0, lo que indica una debilidad en la TD con respecto a la influencia de sus padres. 


\section{Journal of the Academy $|50|$}

El tercer grupo de preguntas corresponde a la TD en situación con los pares. En la situación de apoyar el ir contra de un profesor el ciclo III muestra una media de 1.90. Mientras en el ciclo IV es de 2,27 y en el ciclo $\mathrm{V}$ de 2,44. Esto demuestra que la influencia de los pares disminuye con la edad. Por otro lado, en lo referido a no fumar, el ciclo III muestra fortaleza con una media de 2.6, igualmente el ciclo IV, señala una media de 2.76 y el ciclo $\mathrm{V}$ de 2.80 . Con respecto a la presión de jugar, el ciclo III, presenta una media de 2,67, mientras que el ciclo IV la media es de 2.81 y el ciclo $\mathrm{V}$ es de 2.63. Lo anterior demuestra fortaleza al escoger los deberes académicos sobre el juego. Por último, en la participación en actividades del colegio, el ciclo III muestra una media de 2.03, mientras que en el ciclo IV, es débil con una media de 1.89 y en el ciclo III es de 1.67. Lo anterior indica que los mayores toman decisiones sin interés en la participación de las actividades del colegio.

En el grupo de preguntas que representa los intereses personales, la situación de aceptación de beca en Japón, el ciclo III marca una media de 1.92. En este punto el ciclo IV muestra una media de 2,14 y el ciclo $\mathrm{V}$ de 2,31. Se muestra el aumento en la independencia y asertividad en la TD a medida que aumenta la edad. En el punto sobre tomar decisiones para la comprensión de temas, el ciclo III muestra una media de 2.02; el ciclo IV, de 2.30 y el ciclo V, muestra fortaleza con 2.52. Nuevamente se muestran mejores decisiones en los de mayor edad. En el punto referido al compromiso con la participación en un campeonato, el ciclo III muestra una media de 2.26, el ciclo IV de 2.38 y el ciclo V de 2.27. Se muestra una fortaleza en la TD en todos los ciclos cuando se trata de participación deportiva. Por último, en el proyecto de vida el ciclo III, muestra una media de 2.59, el ciclo IV de 2.77 y el ciclo V de 2,64. Los resultados son positivos en lo que se podría señalar que los estudiantes en los tres ciclos, tienen sueños y piensan en su futuro profesional.

\section{Medición de la Actitud sobre la TD.}

Los resultados obtenidos en los tres ciclos se muestran en la siguiente tabla, donde se perciben posiciones de buena actitud en la TD. 
Journal of the Academy $|51|$

\section{Tabla 5}

Estadística medición de la actitud en la toma de decisiones

\begin{tabular}{|c|c|c|c|c|c|c|c|c|c|c|c|c|}
\hline & \multicolumn{4}{|c|}{ CICLO III } & \multicolumn{5}{|c|}{ CICLO IV } & \multicolumn{3}{|c|}{ CICLO V } \\
\hline Items & 1 & 8 & 10 & 21 & 5 & 10 & 17 & 19 & 21 & 17 & 19 & 21 \\
\hline Válido & 111 & 111 & 111 & 111 & 74 & 74 & 74 & 74 & 74 & 75 & 75 & 75 \\
\hline Perdidos & 0 & 0 & 0 & 0 & 0 & 0 & 0 & 0 & 0 & 0 & 0 & 0 \\
\hline Media & 2,00 & 2,48 & 2,62 & 1,34 & 2,86 & 2,59 & 2,55 & 2,58 & 1,91 & 2,67 & 2,32 & 1,75 \\
\hline Mediana & 2,00 & 3,0 & 3,00 & 1,00 & 3,00 & 3,00 & 2,00 & 3,00 & 2,00 & 3,00 & 2,00 & 2,00 \\
\hline Moda & 3 & 2 & 2 & 0 & 2 & 2 & 2 & 2 & 2 & 2 & 2 & 2 \\
\hline Desv. & 1,41 & 1,24 & ,97 & $\begin{array}{l}1,31 \\
1\end{array}$ & ,956 & ,859 & ,909 & $\begin{array}{l}1,27 \\
1\end{array}$ & $\begin{array}{l}1,21 \\
8\end{array}$ & 1,07 & $\begin{array}{l}1,21 \\
0\end{array}$ & 1,092 \\
\hline
\end{tabular}

Nota: Ítems relevantes en cada ciclo, con respecto a la medición de las medias en actitud. Elaboración propia

\subsection{Medición de la Actitud sobre la TD. Ciclo III}

En la tabla 5, el ciclo III llama la atención la pregunta 1, que señala la consideración de las implicaciones en la TD, muestra una media de 2.0. En el ítem 8, que se refiere a la facilidad en la TD señala una media de 2.48. Posteriormente en el ítem 10, referido a los resultados efectivos en la TC muestra una media de 2.62 y en el ítem 21, que muestra la influencia de los pares en la TD, señala una media de 1.34. En este último, se muestra una debilidad considerable.

\subsection{Medición de la Actitud sobre la TD. Ciclo IV}

La tabla de resultados para ciclo IV, muestra en general datos positivos de una buena actitud en la TD. En lo referido al ítem 5, sobre recomendar a los amigos la TD propias, muestra una media de 2.86. En el 10, que asigna a dar resultados efectivos en la TD señala una media de 2.59. En el ítem 17, en la preferencia a rodearse de personas autónomas, muestra una media de 2.55. En el 19, que señala la interferencia en la TD por parte de otros, muestra una media de 2.58. Y en el ítem 21, que asigna la influencia de los amigos en la TD, muestra una media de 1.91. Éste último, muestra una pequeña mejoría en comparación al ciclo III. 


\section{Journal of the Academy $|52|$}

\subsection{Medición de la Actitud sobre la TD. Ciclo V}

Los resultados del ciclo $\mathrm{V}$, muestran datos positivos con una buena actitud en la TD. Llama la atención las preguntas 17, referida a preferir rodearse de personas autónomas que muestra una media de 2.67. El ítem 19, que se refiere a la interferencia en la TD por parte de otros, muestra una media de 2.32 y el ítem 21, que muestra la influencia de los amigos en la TD, señala una media de 1.75. Lo anterior demuestra una debilidad en la interferencia en la TD por parte de otros y de sus pares. En resumen, los estudiantes prestan atención a las personas que muestran autonomía en la TD, pero no les gusta que interfieran en su Toma de decisiones.

\section{Medición de la Emotividad sobre la TD.}

Los resultados muestran una fortaleza generalizada expresada en la moda de los ciclos. La medición se hizo con respecto a 1. La autonomía, 2. La pasividad, 3. Conciliador, 4. La certeza, 5. La capacidad, 6. La seguridad, 7. La responsabilidad, 8. El ser justo y 9. La generosidad en la toma de decisiones. Se muestran diferencias mínimas al observar los datos de las medias.

\section{Tabla 6}

Estadística medición de la emotividad en la toma de decisiones

\begin{tabular}{lllllllllll}
\hline & & 1 & 2 & 3 & 4 & 5 & 6 & 7 & 8 & 9 \\
\hline C & Válido & 111 & 111 & 111 & 111 & 111 & 111 & 111 & 111 & 111 \\
i & Perdidos & 0 & 0 & 0 & 0 & 0 & 0 & 0 & 0 & 0 \\
c & Media & $3,47^{*}$ & $4,04^{*}$ & $4,14^{*}$ & $4,05^{*}$ & $4,41^{*}$ & 4,33 & 4,11 & 4,23 & 4,39 \\
1 & Mediana & 4,00 & 5,00 & 5,00 & 4,00 & 5,00 & 5,00 & 5,00 & 5,00 & 5,00 \\
o & Moda & 5 & 5 & 5 & 5 & 5 & 5 & 5 & 5 & 5 \\
I & Desv. & 1,482 & 1,220 & 1,132 & 1,115 & 1,074 &, 966 & 1,123 & 1,061 & 1,020 \\
I & & & & & & & & & \\
I & & & & & & & & & & \\
\hline C & Válido & 74 & 74 & 74 & 74 & 74 & 74 & 74 & 74 & 74 \\
i & Perdidos & 0 & 0 & 0 & 0 & 0 & 0 & 0 & 0 & 0 \\
c & Media & $3,34^{*}$ & $3,45^{*}$ & $3,55^{*}$ & $3,45^{*}$ & $4,12^{*}$ & 3,81 & 4,00 & 4,03 & 4,07 \\
1 & Mediana & 3,50 & 3,00 & 4,00 & 3,00 & 5,00 & 4,00 & 4,00 & 4,00 & 4,00 \\
o & Moda & 4 & 3 & 4 & 3 & 5 & 5 & 5 & 5 & 5 \\
I & Desv. & 1,285 & 1,218 & 1,207 & 1,207 & 1,170 & 1,300 & 1,110 & 1,170 & 1,114 \\
V & & & & & & & & & 5
\end{tabular}


Journal of the Academy $|53|$

\begin{tabular}{lllllllllll}
\hline C & Válido & 75 & 75 & 75 & 75 & 75 & 75 & 75 & 75 & 75 \\
i & Perdidos & 0 & 0 & 0 & 0 & 0 & 0 & 0 & 0 & 0 \\
c & Media & $3,43^{*}$ & $3,47^{*}$ & $3,63^{*}$ & $3,57^{*}$ & $4,15^{*}$ & 3,80 & 3,73 & 3,95 & 4,03 \\
1 & Mediana & 3,00 & 3,00 & 4,00 & 4,00 & 4,00 & 4,00 & 4,00 & 4,00 & 4,00 \\
o & Moda & 3 & 3 & 3 & 4 & 5 & 4 & 4 & 4 & 4 \\
V & Desv. & 1,129 & 1,234 & 1,136 & 1,080 & 1,049 & 1,151 & 1,095 & 1,025 & 1,090 \\
\hline
\end{tabular}

Nota: Representan los resultados relevantes en la media sobre emotividad. Elaboración propia

La tabla 6, muestra que en el ciclo III una media en autonomía de 3.47 y en capacidad para TD de 4.41, indicando una diferencia con el ciclo IV, que muestra una media de 3.34 en autonomía y de 4.12 en capacidad para TD. Estos a su vez, muestran un nivel inferior en emotividad con respecto al ciclo $\mathrm{V}$, que tiene una media en autonomía de 3.43 y 4.15 en la capacidad para TD. La diferencia se verifica en el ciclo III, porque se declaran según la media en ser autónomos, activos y conciliadores. Mientras que en ciclo IV según la media se identifican con ser activos, conciliadores y certeros. Igualmente, los estudiantes de ciclo V, se reconocen como activos, conciliadores y certeros. Los buenos resultados en emotividad en el ciclo III llaman la atención puesto que, en este ciclo se presenta mayor grado de reprobación y problemas convivenciales que se verifican por la influencia de los pares y familiares.

\section{Medición de la toma de decisiones según situaciones de estrés, familiar, interés propio y con los pares, entre géneros.}

En cuanto al estrés hay una diferencia a favor del género femenino. Las medias en el género femenino, en las situaciones de estrés son: En peligro, 2.0; en riesgo de perder el trabajo, 2.06; en la prioridad en recuperaciones, 2,57 y en amenazas, es de 2.71. Mientras que el masculino son: En peligro, 2.14; en riesgo de perder el trabajo, 1.70; en la prioridad en recuperaciones, 2.48 y en amenazas es de 2.42. De esta manera las medias indican que las mujeres reconocen las situaciones de riesgo de perder el trabajo, les dan prioridad a las recuperaciones y perciben las amenazas en su contexto.

En las situaciones familiares el género femenino muestra una mejor capacidad de TD demostrado en las medias así: En la escogencia de carrera, 2.63 y al manejo del noviazgo, 2.50. En comparación con los hombres que muestran 2.55 en la escogencia de carrera y 2.36, en el manejo del noviazgo. Las mujeres se muestran un poco distanciadas en cuanto al ser ejemplo 


\section{Journal of the Academy | $54 \mid$}

para sus hermanos, indicando una media de 1.48, mientras los hombres, muestran una media de 1.66

En situaciones de pares, se muestra una ligera diferencia en las medias a favor de las mujeres en situación de presión al fumar, de 2.79; en el cumplimiento con los trabajos escolares de 2.83 y en la participación de actividades en el colegio de 1.95. En este caso de los hombres, en la situación de presión de fumar, de 2.60; en el cumplimiento con los trabajos escolares de 2.55 y en la participación de actividades en el colegio de 1.81 .

En los intereses propios, las mujeres toman mejores decisiones representados en las medias del aprovechamiento de becas de estudio con 2.11 y los hombres con 2.07. En la búsqueda de comprensión de temas de estudio, las mujeres tienen una media de 2.32 y los hombres de 2.15. Los hombres en situación de campeonatos, presentan una media de 2.36 y a las mujeres que muestran, 2.24. En el aspecto de proyecto de vida frente a la TD, no hay diferencia significativa entre ambos géneros.

\section{Medición de la actitud por género.}

En las actitudes los hombres muestran una ligera ventaja respectivamente en las medias significativas así: Las implicaciones en la TD de 2.36; la capacidad de actuar en la TD, 2.93; la facilidad para TD, 2.76 y la toma de conciencia al TD de 3.10. Mientras las mujeres poseen sobre medias en las implicaciones en la TD, 2.09; la capacidad de actuar en la TD, 2.77; la facilidad para TD, 2.67 y la toma de conciencia al TD, 3.07. Los hombres muestran una ligera ventaja demostrado en las medias, una mayor confianza en la TD. 


\section{Journal of the Academy $|55|$}

\section{Correlaciones de Pearson por género.}

\section{Tabla 7.}

Actitudes de los hombres y las mujeres frente a la TD

\begin{tabular}{|c|c|c|c|c|c|}
\hline & & \multicolumn{2}{|l|}{ Hombres $\mathrm{N}=124$} & \multicolumn{2}{|l|}{ Mujeres $\mathrm{N}=136$} \\
\hline & & $\begin{array}{l}\text { Capacidad de } \\
\text { actuar según la } \\
\text { situación }\end{array}$ & $\begin{array}{l}\text { Tiene } \\
\text { facilidad } \\
\text { para TD }\end{array}$ & $\begin{array}{l}\text { Capacidad de } \\
\text { actuar según la } \\
\text { situación }\end{array}$ & $\begin{array}{l}\text { Tiene } \\
\text { facilidad } \\
\text { para TD }\end{array}$ \\
\hline ¿Cuál es & Correlación de &.$^{\mathrm{a}}$ &.$^{\mathrm{a}}$ &. $\mathrm{a}$ &. $\mathrm{a}$ \\
\hline Género? & Pearson & & & & \\
\hline & Sig.(bilateral) & . & . & . & . \\
\hline ¿Considera las & Correlación de &, 129 &, 065 &, $178 *$ &, 120 \\
\hline implicaciones de & Pearson & & & & \\
\hline la TD? & Sig. (bilateral) &, 153 & ,473 & ,038 &, 163 \\
\hline ¿Se cree capaz de & Correlación de &, $468^{* *}$ &, $440^{* *}$ &, $322 * *$ &, $330 * *$ \\
\hline tomar sus & Pearson & & & & \\
\hline decisiones? & Sig. (bilateral) &, 000 &, 000 & ,000 & ,000 \\
\hline
\end{tabular}

Nota: a. No se puede calcular porque, como mínimo, una de las variables es constante *. La correlación es significativa en el nivel 0,05 (bilateral). **. La correlación es significativa en el nivel 0,01 (bilateral).

En la tabla 7, las correlaciones significativas aparecen las mujeres entre la capacidad de TD y la capacidad de actuar, con 0.322 y los hombres, 0.468. Las mujeres muestran entre la capacidad y la facilidad de TD con 0.330 y los hombres, 0.440. Las mujeres, muestran entre las implicaciones y la capacidad de actuar, 0.178 y los hombres, 0.129. Así, los hombres toman decisiones rápidas y las mujeres, toman más tiempo en la TD, pero se muestran más certeras en su decisión.

\section{Correlación de Pearson.}

Evalúa las actitudes como variables dependientes según la edad, el ciclo y género. 


\section{Journal of the Academy $|56|$}

\section{Tabla 8}

Correlación de regresión Pearson

\begin{tabular}{llll}
\hline Variable dependiente & Variable independiente & $\mathrm{R}$ & $\mathrm{R}^{2}$ \\
\hline Tomar en cuenta las consecuencias Genero 1 & Edad y Ciclo & $0.057^{*}$ & 0.003 \\
Tomar en cuenta las consecuencias Genero 2 & Género & $0.011^{*}$ & 0.000 \\
Capacidad de actuar Género 1 & Edad y Ciclo & $0.196^{*}$ & 0.038 \\
Capacidad de actuar Género 2 & Género & $0.069^{*}$ & 0.005 \\
Facilidad para TD Género 1 & Edad y Ciclo & $0.189^{*}$ & 0.036 \\
Facilidad para TD Género 2 & Género & $0.007^{*}$ & 0.000 \\
\hline
\end{tabular}

Nota: *resultados de correlación positivos y cercanos a 0. Elaboración propia

La tabla 8 muestra en los resultados actitudinales de regresión, valores en $\mathrm{R}$ positivos muy bajos. Estos valores bajos, señalan una pequeña dependencia entre edad, ciclo y género. Para ello, se debe tener en cuenta, las otras relaciones sobre los ítems asignados a las subcategorías de conocimiento y emotividad.

\subsection{Medición de la emotividad por género.}

Con respecto a la parte emotiva se encontraron datos positivos en ambos géneros. Sin embargo, en los hombres se verifica una pequeña ventaja en las siguientes medias: Ser conciliadores, 3.86; ser capaces en la TD, 4.10 y por último en ser seguros y autónomos en la TD, 3.48. En las mujeres, se muestra en ser conciliadoras, 3.79; ser capaces en la TD, 3.97 y en ser seguras y autónomas en la TD, 3.36. Hay que señalar que existe una correlación considerable en las mujeres en la actuación al tomar decisiones y la consideración de consecuencias. 
Journal of the Academy | $57 \mid$

\section{DISCUSIÓN DE RESULTADOS}

Comparando los resultados obtenidos en el estudio con los porcentajes de reprobación y deserción 2017 y 2018, (ver tabla 9), encontramos lo siguiente:

\section{Tabla 9}

Estadística de reprobación y deserción 2017-2018

\begin{tabular}{|l|l|l|l|l|}
\hline \multicolumn{2}{|l|}{2017} & 2018 \\
\hline & REPROBACIÓN & DESERCIÓN & REPROBACIÓN & DESERCIÓN \\
\hline CICLO III & $57.8 \%$ & $62.1 \%$ & $37.19 \%$. & $45.7 \%$ \\
\hline CICLO IV & $28.07 \%$ & $27.07 \%$ & $38.01 \%$ & $29.6 \%$ \\
\hline CICLO V & $14.03 \%$ & $10.8 \%$ & $24.79 \%$ & $24.69 \%$ \\
\hline
\end{tabular}

Nota: Estadísticas, resultados de reprobación y deserción, según coordinación secundaria jornada tarde.

Elaboración propia

La comparación de la TD según el ciclo con los resultados académicos y convivenciales, demuestra una relación directa fundamentada en la edad y el ciclo al cual pertenece. Lo anterior, se evidencia en el alto porcentaje de reprobación y deserción que se presenta en el ciclo III y que disminuye en el ciclo cuatro y cinco. Esta relación se convierte en un reto para fortalecer la TD en ciclo III.

En otros resultados, la convivencia se presenta en datos suministrados por coordinación y se muestran en porcentajes de agresiones verbales, físicas, evasiones de clase y hurtos. Dicha problemática, disminuye al ir aumentando en edad y en los ciclos. Esto permite la explicación fundamentada en el grado de madurez y aprendizaje individual, al ser más conscientes que la TD afecta las metas propuestas para su proyecto de vida (Gallego, 2015). Consecuentemente, permite una evolución desde la dependencia de las familias y los pares, hasta los propios intereses. Todo, se afianza en la influencia de las experiencias vividas. Igualmente, los resultados obtenidos, refuerzan los señalamientos de Gallego (2015), con respecto a la importancia de los espacios familiares y escolares en la primera infancia, que forman vínculos 


\section{Journal of the Academy $|58|$}

en el intercambio de ideas y las formas de ser y actuar. También, interviene el diálogo y el acercamiento con los padres y los pares a través de las vivencias. González, Carrillo y Palma (2017).

Al comparar los datos obtenidos entre los tres ciclos, se establece un fortalecimiento en la TD al ir independizando su habilidad con respecto a la familia y a los pares ya que, no admiten la interferencia de otros en la TD. Finalmente, se fortalece la TD con respecto al interés personal y a la responsabilidad en los deberes escolares. Lo anterior, concuerda con los estudios de Bonilla, Bonilla, Muñoz y Baldovino (2017) y González, Orcasita; Carrillo; y Palma, (2017), cuando observan el incremento en la calidad de la habilidad al aumentar la edad.

En las actitudes se muestra una influencia de los pares en la TD demostrando la interacción social que puede ser negativa o positiva. En este punto, Enciso y Lozano, (2011), señalan la importancia de las competencias sociales desde la formación de conductas que son aceptadas y valoradas por la sociedad. De esta manera, el aprendizaje de la TD se orienta hacia el bienestar particular y grupal.

En otro aspecto, el presente estudio es consecuente con Enciso y Lozano (2011), al mostrar que la habilidad de TD se puede analizar desde lo emocional, lo conductual y lo cognitivo. A su vez, se pueden fortalecer a través del aprendizaje y el entrenamiento.

Los datos obtenidos concuerdan con Mora, Martínez y Serrano (2013), al mostrar la influencia del estrés, la familia, los pares y los intereses personales en la TD. En el estudio, se evidenció el efecto de las emociones en la TD, ya que depende de experiencias vividas que se insertan en el proceso cognitivo del sujeto.

Comparando resultados entre los hombres y mujeres, encontramos que las mujeres tienen una relativa ventaja al ser más prudentes al tomar decisiones por las influencias de índole familiar, los pares, el estrés y el interés propio. Lo anterior, concuerda con Cote y García (2016) en la actitud por género, en tanto, el nivel de confianza implica que los hombres tienden a ser más ligeros en la TD.

Finalmente, para hacer válido el estudio, se realizan las respectivas correlaciones entre las actitudes encontrándose una correlación directa y válida entre los ítems. 


\section{Journal of the Academy | 59 |}

\section{CONCLUSIONES}

Las habilidades para la vida se consideran como habilidades psicosociales que permiten la interacción sana entre las personas y que cada persona, posea herramientas para enfrentarse de manera exitosa a los desafíos de la vida cotidiana. Dentro de estas habilidades, la TD tiene correlación directa con otras habilidades como la comunicación, el asertividad y el autocontrol. Ésta a su vez, se desarrolla y se fortalece con la edad, con el contexto y las experiencias vividas.

Al ubicar la muestra en el contexto y visualizando la caracterización socioeconómica de los estudiantes, encontramos que se ubican en estratos uno, dos y tres. También, que gran porcentaje de los padres, no poseen estudios técnicos o universitarios y que su sustento económico depende de la economía informal. Esto redunda en el porcentaje de deserción desde la decisión de índole familiar. En consecuencia, cuando los estudiantes dependen de las familias y en sus pares, en la TD, los estudiantes muestran debilidad en la habilidad. Los resultados en el desarrollo de recuperaciones y deberes académicos, muestran un incremento positivo al avanzar en los ciclos, cuando manifiestan mayor responsabilidad en la TD. Igualmente, los resultados señalan que no les gusta la interferencia de otros en su TD, al incrementar del ciclo III al ciclo V.

Para la caracterización, se tiene en cuenta que los cursos superiores muestran resultados satisfactorios en la TD concordando con los resultados estadísticos de reprobación y deserción de la oficina de coordinación. Esto, no quiere decir que la TD esté fortalecida en los ciclos superiores, pues, se considera que, aunque la correlación entre las actitudes es positiva, éstas no explican los resultados negativos académicos y convivenciales de los años 2017 y 2018 . En consecuencia, la caracterización indica que la habilidad de TD debe ser mejorada con la conjugación de otras habilidades como la asertividad, la comunicación, el ser críticos y tolerancia entre otras.

El presente estudio, tuvo limitaciones que se desprenden desde el método utilizado para identificar las categorías que señalan los factores que influyen en la TD, este método, se basó en la observación y en los datos obtenidos a través de entrevistas informales a estudiantes, padres y profesores, durante los años 2017 y 2018. Lo anterior, pudo haber dejado otros aspectos de influencia que no se identificaron en el desarrollo de las observaciones y entrevistas. Por otro lado, el 100\% de la población de estudio, no posee la aplicación de un 


\section{Journal of the Academy $|60|$}

programa que trabaje las habilidades para la vida, como proyecto derivado del Plan Educativo Institucional, PEI.

Para finalizar, los resultados abren la posibilidad de nuevos estudios que logren plantear el fortalecimiento de la TD, teniendo en cuenta proyectos o programas que se desarrollan en el colegio, igualmente, desarrollar un estudio de carácter cualitativo, que identifique por parte de padres, estudiantes y profesores, la posibilidad de integrar en el currículo, el conocimiento y las acciones, que permitan que la habilidad de la TD se fortalezca de manera responsable favoreciendo la salud y el bienestar de la comunidad.

\section{REFERENCIAS BIBLIOGRÁFICAS}

Aranguren, M. (2015). Influencia del conocimiento previo sobre el test de pensamiento creativo de Torrance. International Journal of Psychological research, 8(2): 75-89. Recuperado de http://www.scielo.org.co/pdf/ijpr/v8n2/v8n2a07.pdf

Barbosa, V., Pereira, Z., y Kopller, A. (2015). Habilidades Sociales y el Modelo Bioecológico de Desarrollo Humano: análisis y perspectivas. Psychology, Society \& Education, 28(1): 181-193. doi: http://dx.doi.org/10.1590/1807-03102015aop001.

Barrantes, L. (2016). Educación emocional: El elemento perdido de la justicia social. Educare, 20(2): 1-10. doi: http://dx.doi.org/10.15359/ree.20-2.24

Bonilla, J., González, A., Bonilla, G., Muñoz, F., Baldovino, E. (2017). Toma de decisiones en menores con factores de riesgo para el Trastorno Disocial de la Conducta. Revista médica Risaralda, $23(1)$ : 13-16. $\quad$ Recuperado de http://www.scielo.org.co/scielo.php?pid=S0122$\underline{06672017000100004 \& \text { script }=\text { sci_abstract\&tlng=es }}$

Choque, R., y Chirinos, J. L. (2009) Eficacia del Programa de Habilidades para la Vida en Adolescentes Escolares de Huancavelica, Perú. Revista de Salud Pública Salud pública, 11(2): 169-181. Recuperado de http://www.scielo.org.co/pdf/rsap/v11n2/v11n1a02.pdf

Cote, L. P., y García, A. M. (2016). Estrés como factor limitante en el proceso de toma de decisiones: una revisión desde las diferencias de género. Avances en Psicología. Latinoamericana, 34 (1): 19-28. doi: http://dx.doi.org/10.12804/apl34.1.2016.02

Decreto 1860 de 1994 Educación Ministerio de Educación Nacional de Colombia. Recuperado de: https://www.mineducacion.gov.co/1759/articles-86240_archivo_pdf.pdf

Di Domenico, J. B., y Scortegagna, A. (2018). Validade Convergente do Zulliger-SC con Inventário of Habilidades Sociais para Idosos. Paidéia (Ribeirão Preto), 28 (e 2832): 110 doi: http://dx.doi.org/10.1590/1982-4327e2832

Enciso, E. y , Lozano, M. (2011). Diferencias en actitudes y estrategias cognitivas sociales en jóvenes vinculados y no vinculados a programas de voluntariado. Psychologia. Avances 


\section{Journal of the Academy $|61|$}

de la disciplina, 5(2): 81- 95. Recuperado de: http://www.scielo.org.co/scielo.php?script=sci_abstract\&pid=S1900$\underline{23862011000200007 \& \operatorname{lng}=\text { es\&nrm=iso }}$

Gallego, A. (2015) Concepciones adultas sobre participación infantil en relación con la toma de decisiones de los niños. Zona Próxima, 22, 87-104. doi: http://dx.doi.org/10.14482/zp.22.6078

García, B. (2009). Manual de métodos de investigación para las ciencias sociales. Un enfoque de enseñanza basado en proyectos. Editorial El Manual Moderno S.A. de C.V, México D.F

González, Claudia., Guevara, Y., Jiménez, D., Alcázar, R. (2018). Relationship between Assertiveness, Academic Performance and Anxiety in a Sample of Mexican Students in Secondary Education. Act.Colom.Psicol, 21 (1): 116-127. doi: http://dx.doi.org/10.14718/acp.2018.21.1.6

González, V., Orcasita, T., Carrillo, J. P., y Palma, D. M. (2017). Comunicación familiar y toma de decisiones en sexualidad entre ascendientes y adolescentes. Revista Latinoamericana de Ciencias Sociales, Niñez y Juventud, 15(1): 419-430. doi: http://dx.doi.org/10.11600/1692715x.1512605022016.

Gualano de Godoy, M. (2011) La Democracia Deliberativa como Guía para la Toma de Decisiones Legítimas. Análisis teórico a partir de Carlos Santiago Nino y algunas prácticas institucionales en el Brasil contemporáneo. Co-herencia, 8 (14): 63-91. Recuperado de http://www.scielo.org.co/pdf/cohe/v8n14/v8n14a03.pdf

Hernández, R., Fernández, C. y Baptista P. (2014). Metodología de la investigación. 6ta edición. Mc Graw-Hill / Interamericana Editores, S.A, México D.F.5

Lastra, S. P., Durán, N. C.,\& Acosta, D. (2018). Community based pedagogy as an eye-opening for pre-service teachers' initial connections with the school currículo. Colombian Applied Lingüistic Journal, $\quad 20(2): \quad 196-209 . \quad$ doi: http://dx.doi.org/10.14483/22487085.13047

Ley 115 de 1994 Ley general de Educación Ministerio de Educación Nacional de Colombia. Recuperado de: https://www.mineducacion.gov.co/1621/articles$\underline{85906 \text { archivo pdf.pdf }}$

Ley 1620 de 2013. Sistema Nacional de Convivencia Escolar y formación para el ejercicio de los Derechos Humanos, la Educación para la Sexualidad y la Prevención y Mitigación de la Violencia Escolar. Recuperado de: https://www.mineducacion.gov.co/1759/articles327397_archivo_pdf_proyecto_decreto.pdf

Márquez-Cervantes, C., Gaeta-González, M. (2018). Competencias emocionales y toma de decisiones responsable en preadolescentes con el apoyo de docentes, padres y madres de familia: Un estudio comparativo en estudiantes de $4^{\circ}$ a $6^{\circ}$ año de educación primaria en España. Educare, 22(1): 1-25. doi: http://dx.doi.org/10.15359/ree.22-1.9 


\section{Journal of the Academy | $62 \mid$}

Martínez, J. W., Tovar, J. R., Rojas, C., y Duque, A. (2008). Agresividad en los escolares y su relación con las normas familiares. rev.colomb.psiquiatr, 37(3): 365-377. Recuperado de: http://www.scielo.org.co/pdf/rcp/v37n3/v37n3a07.pdf

Martínez, R. V. (2014) Habilidades para la vida: una propuesta de formación humana. Itinerario Educativo, 28 (63): 61-89. doi: https://doi.org/10.21500/01212753.1488

Mora, F., Martínez, J., Serrano, G. (2013). Los factores individuales y su efecto en los resultados escolares. Una medición a partir del SINCE TIC 2013. Calidad en la educación, 48, 130-165. doi: http://dx.doi.org/10.31619/caledu.n48.481

Moscoso, M. S., y Spielberger, C. D. (2011). Evaluación intercultural de las emociones: la expresión de la ira. Revista de Psicología, 29 (2): 343-360. doi: http://revistas.pucp.edu.pe/index.php/psicologia/article/view/2472

Mosqueda, A., Mendoza, S., Jofré, V., y Barriga, O (2015). Validez y confiabilidad de una escala de apoyo social percibido en población adolescente. Enfermería Global, 14(39): 125-136 Recuperado de http://scielo.isciii.es/scielo.php?script=sci_arttext\&pid=S1695$\underline{61412015000300006}$

Ospina, B. E., Sandoval, J. J., Aristizábal, C. A., Ramírez, M. C., (2005) La escala de Likert en la valoración de los conocimientos y las actitudes de los profesionales de enfermería en el cuidado de la salud. Investigación y Educación en Enfermería, 23(1): 14-29 Recuperado de $\quad$ http://www.scielo.org.co/scielo.php?pid=S012053072005000100002\&script=sci_abstract\&tlng=es

Portillo, M. C. (2017). Educación por habilidades: Perspectivas y retos para el sistema educativo. Revista Educación, http://dx.doi.org/10.15517/revedu.v41i2.21719

Vargas, E., Henao, J., González, C. (2007). Toma de decisiones sexuales y reproductivas en la adolescencia. Acta Colombiana de Psicología, 10(1), 49-63. Recuperado de http://www.scielo.org.co/scielo.php?pid=S0123-

91552007000100006\&script=sci_abstract\&tlng=es

Veloso, V., Magalhães, H., Vasconcelos, I. C., Costa, M. G., Teixeira, A., y Rayane, T. (2018). Emotional Regulation Questionnaire (ERQ): Evidence of Construct Validity and Internan Consistency. Psico-USF, 23(3): 461-471. doi: http://dx.doi.org/10.1590/141382712018230306

Ventura, J., Caycho, T (2016). Análisis psicométrico de una escala de dependencia emocional en universitarios peruanos. Revista de psicología (Santiago), 25(1): 1-17. doi: http://dx.doi.org/10.5354/0719-0581.2016.42453

Vieira, N., Barbosa, V., Goncalves, M. R. (2018). Influence of Social Skills and Stressors on Academic Achievement in the Sixth-Grade. Paideia (Ribeirão Preto), 28, (e 2819). doi: http://dx.doi.org/10.1590/1982-4327e2819 